\section{ESNR New Year editorial}

\author{
Johan Van Goethem ${ }^{1,2}$
}

Published online: 15 December 2019

(C) Springer-Verlag GmbH Germany, part of Springer Nature 2019

\section{When will neuroradiologists be replaced by robots?}

\section{Not all image processing software is artificial intelligence}

Artificial intelligence (AI) is a buzz word used and misused not only by the popular press but also in neuroradiology. You may find it hard to believe but not every software tool you use today that does some fancy stuff with your images is AI: in fact, far from it! Most of these tools are actually just "plain software." For example, do you have software that automatically numbers your spinal levels, aligns your MR-scans to the patient's anatomy, or a tool that calculates brain volumes? If so, you might simply be using clever software programmed by clever people with many pre-programmed steps dictating how to perform specific actions, rather than utilizing AI.

So what is AI? AI is software, but it is not pre-programmed to solve all problems at hand or specific problems with multiple details. Instead, AI knows more or less what to do but in the beginning is not very good at anything. Yet, AI has the capacity to change the way it works and to process data in different ways; in other words, AI can reprogram itself to "get better," essentially having the ability to "learn." How does that work? Well, let us say AI is supposed to find fractures on CT images. However, it has no clue what to do with the images or what a fracture looks like! This is where the specialist comes in, a smart person, i.e., a neuroradiologist. The neuroradiologist tells the software where the fractures are and the AI digs in the voxels to find a rule that defines a fracture. In the beginning, the AI is worse than any radiologist, than any medical student, and hey, pretty much

Johan Van Goethem

johan.vangoethem@uantwerpen.be

Department of Radiology, AZ Nikolaas, Sint-Niklaas, Belgium

2 Neuroimaging and Biomedical Imaging Techniques, University of Antwerp, Antwerp, Belgium worse than anyone! But each time the neuroradiologist tells AI which fractures it correctly or incorrectly diagnoses, the AI software digs into the bits and bytes (we call them voxels) of the image data again and again, and with every step it implements algorithm changes to provide the best result. Bingo! We have machine learning! In machine learning, programmers build a mathematical model based on sample data - known as "training data" - in order to make predictions or decisions without being explicitly programmed to perform the task [1].

Keep in mind that this algorithm does not work to detect hypo-intense lines (fractures) in hyperdense stuff (bone) - at least not exactly. In fact, AI might look at things neuroradiologists do not look at or cannot look at such as finding every $\mathrm{x}$ number of voxels that form a line with densities within a certain threshold. After finding all of these voxels in the whole data set, AI might check which ones are within a certain distance of each other or which ones are oriented in certain 3D angles. Importantly, AI can continually carry out and look for changes in consecutive steps and build many small steps in succession, with the additional advantage that every step can be more or less freely changed. Bingo 2 ! We now have an artificial neural network (ANN)! In the end, no one knows, or at least no one needs to know, how AI finds the fractures: it just works like magic! An ANN is based on a collection of connected units or nodes called artificial neurons, which loosely model biological brain neurons. Each connection, like biological brain synapses, can transmit a signal to other neurons. An artificial neuron that receives a signal then processes it and can signal neurons connected to it [2]. Finally, AANs allow for something called deep learning. Deep learning is a subfield of machine learning concerned with algorithms inspired by the structure and function of the brain called artificial neural networks [3]. Are you now confused? Read on. Deep learning is an AAN on steroids. The enormous computer power available today allows for very large AANs to work on very large unlabeled datasets (no need to point out where the fractures are), to work with analog datasets (typically images/ video), and to do feature extraction on these images. Feature extraction works in layers, e.g., finding brain and skull 
contours first, then recognizing lobes and ventricles, as well as gray and white matter, subsequently determining lesion locations and finally characterizing these lesions and suggesting a diagnosis. Most AI in neuroradiology today uses machine learning, ANNs, and deep learning to some extent.

\section{The problem with AI}

Tesla promised "Full Self-Driving Capability" in 2016. Three years later it remains in the "unknown future activation" stage. To understand why, let us go back. The Society of Automotive Engineers defines five levels of self-driving cars. Level 1 is the good old cruise control with added radar which can keep one car a predefined distance from the car in front. This technology has been around for more than 10 years. Add steering to keep a car in a motorway lane and you achieve Level 2, offered by many modern cars (usually as an expensive option). Now the critical part: Level 3 cars can take care of all aspects of the dynamic driving task, but the human driver needs to respond appropriately to intervention requests. As of today, no Level 3 cars exist (neither do Level 4 or Level 5 cars, the latter being as performant as a human driver). In 2017, Audi announced that its new flagship A8 would be a Level 3 self-driving car when it hits the road in ... 2019 [4]. The car has arrived with all the necessary hard- and software, but the feature is not enabled. Why? It comes down to a vast array of regulatory, technical, safety, and legal and business-related complications, which has brought the car to a halt. One major issue is that of responsibility: Who will be responsible when something goes wrong? The car manufacturer? The supervising driver? In essence, the handover from software to human does not always go well, as experiments with Level 3 self-driving cars have revealed. But Level 4 or Level 5 cars are not there either. With Level 4 and 5 cars, AI should theoretically be able to handle all worldly situations safely: heavy snow, bright sunshine, roadworks, double lane markings in construction areas and foolish drivers. But AI should also be able to handle unexpected pedestrians (children!), fallen trees, potholes, ghost drivers, flat tires, and meteor impacts (joke!). You begin to see the problem. A real Level 5 self-driving car would need to know how the world works! For instance, in 2017 a self-driving bus in Las Vegas was involved in an accident on its first day because the self-driving vehicle did not respond while a truck backed up, albeit slowly, towards the bus. The AI controlling the bus, contrary to what a human would do, simply remained immobile until it was hit by the truck. And last year, unfortunately, the first recorded case of a pedestrian fatality involving a self-driving car occurred when an Uber self-driving vehicle did not see nor respond to a pedestrian crossing the road.

\section{Al in neuroradiology}

Commercially available AI-packages are capable of amazing actions: bleed detection, determining core and penumbra of an infarct, comparing lesion loads on sequential MRIs of MS patients, calculating gray matter volume of all the different brain lobes (and the hippocampi and assess whether normal for age) and there are even packages available that will give you the differential diagnosis of brain masses. Since some of these tasks are not always feasible for a neuroradiologist, they provide new probes, as in new instruments. AI may provide new ways to extract information out of neuroimaging datasets, and at other times, AI may try to mimic the way neuroradiologists work only doing it better or faster.

\section{Al will not replace neuroradiologists}

The way AI is used to simulate human reasoning in neuroradiology today makes for a system that is brilliant one day and utterly stupid another day. For example, AI can find subtle bleeds that may be missed by radiologists when looking for brain hemorrhage, especially in high workload environments where we all might miss a small high density subdural if we do not window images optimally. In these situations, an AI backup is great. Nevertheless, in other cases, the computer might flag areas as hemorrhage when it is obvious to neuroradiologists that the machine defined "hemorrhages" are artifacts, bone or other matter, which may encourage referring physicians to accept an incorrect AI diagnosis as they may lack the necessary experience to make a correct diagnosis. For this reason, it's likely that a supervising neuroradiologist will still be needed for at least a decade to ensure correct diagnoses. In short, we can compare our current situation to that of a Level 2 self-driving car: AI helps the radiologist but the radiologist is still required at all times.

\section{On the contrary, Al increases the need for neuroradiologists}

In no uncertain terms, AI tools make neuroradiology more complex, especially since the tools require human supervision much in the way Level 3 self-driving cars do. As doctors, we will always be responsible for our final reports and our diagnoses. We need to integrate all of these fabulous new tools into our daily practice. And for clinicians, neuroradiology will only get more overwhelming. Saying that, there is good newsyou can sleep on both ears for neuroradiologists are here to stay. At least for the next decade!

Have a Happy 2020!

Johan Van Goethem

ESNR President 


\section{References}

1. Bishop CM (2006) Pattern recognition and machine learning, Springer ISBN 978--0--387-31,073-2

2. Artificial neural network in Wikipedia. Retrieved October 19, 2019, from https://en.wikipedia.org/wiki/Artificial_neural_network. Accessed 26 Oct 2019

3. Brownlee J (2019) Deep learning \& artificial neural networks in deep learning
4. The new Audi A8 - conditional automated at level 3, 09/11/17. Retrieved October 19, 2019, from https://www.audi-mediacenter. com/en/on-autopilot-into-the-future-the-audi-vision-of-autonomousdriving-9305/the-new-audi-a8-conditional-automated-at-level-39307. Accessed 26 Oct 2019

Publisher's note Springer Nature remains neutral with regard to jurisdictional claims in published maps and institutional affiliations. 